\section{Investigating the Microstructure of a Newly Developed Aluminum Alloy Through X-ray Microanalysis}

\author{
Patrick Camus and David Rohde \\ Thermo Electron Corporation, Madison, WI \\ david.rohde@thermo.com
}

\section{Introduction}

The distribution of components, or microstructure, within a material is critical to its mechanical properties. For example, if a material has too many particles of one particular phase it may become too brittle, yet if there are too few particles it could lose strength. Every material exhibits different and unique characteristics. The goal of a material scientist is to analyze a material's microstructure and to optimize the manufacturing process. Electron microscopists observe this distribution on a very small, but critical, scale. The electron microscope can demonstrate specifically where the particles are distributed and then X-ray microanalysis can be used to identify what the particles are composed of.

This article will discuss the microstructure of an aluminum alloy with copper and iron additions. At the point of development, the mechanical properties of this new alloy were believed to be superior to that of previous materials; however, a number of subsequently conducted heat treatments did not support this theory, producing extremely unsatisfactory properties. In order to understand these results and rectify any problems, X-ray microanalysis was used to analyze the alloy's microstructure and assess the distribution of copper and iron particles within the metal.

\section{X-ray Microanalysis}

$\mathrm{X}$-ray microanalysis is one of the most powerful and popular methods available to today's microscopists for the chemical analysis of samples. When the beam of an electron microscope strikes a specimen its electronic structure undergoes changes resulting in the emission of X-rays that are specific for the elements present in the sample. These X-rays can be analyzed by either energy-dispersive or wavelength dispersive spectrometers, to yield a graph or map of the amount and distribution of the elements in the sample.

$\mathrm{X}$-ray microanalysis provides a new piece of chemical information to data collected on electron microscopes. It also uses the spatial knowledge from the microscopic image to provide a spatial component to the data, allowing the distribution of elements to become visible.

A primary concern for many electron microscopists using $\mathrm{X}$ ray microanalysis, however, is understanding the spatial distribution of elements within a sample. With traditional elemental mapping techniques, it is necessary to be aware of all the elements of interest that exist in a sample before the data collection begins. However, this is not always possible and if an element is not expected but is present, a 'black hole' may appear in the maps at its location, or the element may go totally unnoticed.

For this application a Scanning Electron Microscope (SEM) was used to view aluminum alloy samples, after they had been mounted and polished. The SEM shows very detailed 3-dimensional images at much higher magnifications than is possible with a light microscope.
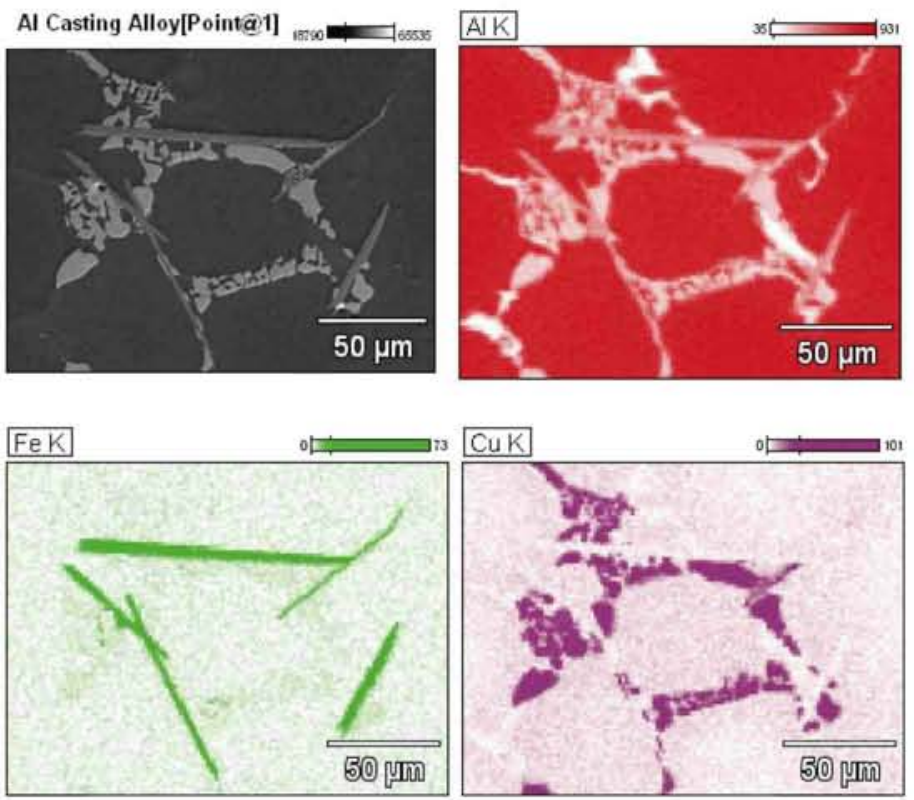

Figure 1 - Electron Image and X-ray maps of casting alloy

\section{Spectral Imaging}

Recent developments have seen the introduction of Spectral Imaging for data acquisition. Instead of collecting a single spectrum at a single location or collecting maps of previously identified elements. Spectral Imaging collects a complete spectrum at every location within a mapped area. This acquisition thus creates a data-cube of X-ray intensity information with the axes being $(x, y)$ locations and energy of the X-rays.

Spectral Imaging reduces problems experienced by previous techniques as it can collect a complete spectrum at each pixel location, making prior knowledge of the presence of elements purely optional. This is made possible by the ability to query the stored data-cube after its collection, allowing elemental map extraction of any unexpected elements days or even years after the initial acquisition. Spectral Imaging data combines elemental and spatial data to create an ideal representation of a sample, seemingly completing the analysis process by providing a thorough understanding of the structure of the material. However, it is possible to gain an even deeper understanding of data than by the use of familiar tools for spectral analysis, such as X-ray mapping or linescans.

The automated statistical analysis tool used to analyze the aluminum alloy was COMPASS and the distribution software was XPhase, both of which are features of Thermo Electron's X-ray microanalysis system NORAN System SIX. These features provide the analyst with the next important level of information: compound identification. Elements do not usually exist in isolation; they tend to form compounds or mixtures with other elements known as phases.

COMPASS provides a method for analyzing Spectral Imaging data sets that analyzes the complete data-cube and compares every spectrum to every other. As this automated statistical analysis tool does not have a bias about which elements to expect in a data set, it will find all components (element mixtures) that are statistically significant, even if they are unexpected to the user. The automated statistical analysis quickly generates "pure" component maps with single-pixel sensitivity and completely eliminates time-consuming 


\section{W" Omniprobe Short-Eut ${ }^{\text {Tw }}$ brings HIHI THPOUHPUT to in-situ TEM sample preparation}

In-Situ FIB Lift-Out

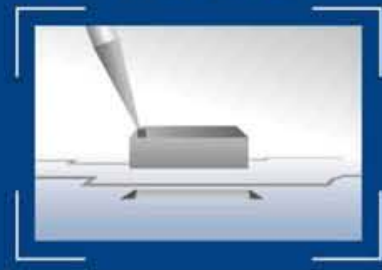

Streamlined in-situ

FIB lift-out with

Omniprobe's

patented Total

Release $^{\mathrm{TM}}$

technology

Mechanical Conversion

गागाग

TEM Sample Prep And Nano-Mechanical Testing

The AutoProbe ${ }^{\mathrm{TM}} 200$ is a computer controlled in-situ multipurpose nanomanipulator system capable of:

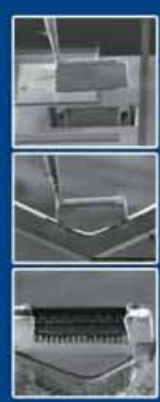

- Lift-out for TEM Sample Prep

- Electrical Measurements

- Nano-Mechanical Testing Ideal For:

- IC Process Control and

Failure Analyses

- Topographic Surfaces

- Brittle or Soft Materials

- Phase Boundaries

- MEMS Components

C $\epsilon$ and UL certified

Patented Technology

AutoProhe 200
Omniprobe, Inc. 10410 Miller Road Dallas, TX 75238 Phone: 214-572-6800

Fax: 214-572-6801

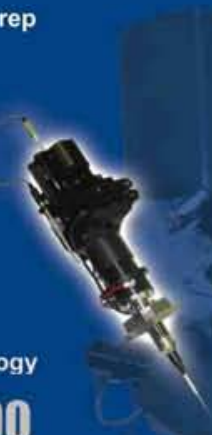

\section{Computer Controlled Multi-Gas} Injection System

The OmniGIS ${ }^{T M}$ is Omniprobe's new multiple gas injection system for the FIB or SEM. The OmniGIS ${ }^{\text {TM }}$ provides programmable control of three gas sources and an additional purge/carrier gas source.

Programmable Process Flow

- 3 Independent Gas Sources

- User-Replaceable Crucibles

- Feedback Control

- Patent Pending

\section{OmnitHSт'}

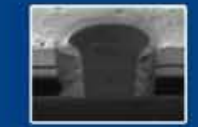

Multiple Gas Sources (Oxide Isolated PI Via) conversion from lift-out to TEM sample 


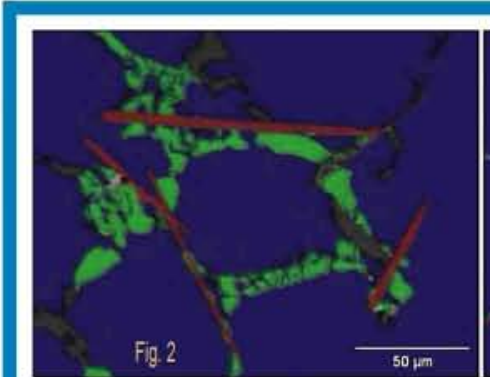

Figure 2 - Expected components (aluminum, copperenriched, iron-enriched).

point-by-point investigations.

The distribution software extends the analytical capabilities of SEM-EDS by extracting elemental phase information from EDS data, providing a complete understanding of the phase distribution in a sample. The software also equips the user with the ability to analyze both X-ray maps and component maps, defining the true chemical localization from Spectral Imaging data, phase maps and extracted phase spectra. Xphase software can find locations within a collection of maps that form uniquely defined compound phases.

\section{Results}

As copper and iron are relatively low- to mid-periodic table elements, a beam voltage of about $15 \mathrm{kV}$ was necessary to detect all the elements. Spectral Imaging data was collected for all X-rays up to $10 \mathrm{kV}$ at a pixel resolution of $128 \times 96$. Aluminum, copper and iron were all selected for map display because they were known to exist in the sample (figure 1). The iron particles were found to be whisker-shaped while the copper particles more globular-shaped.

Previously, the analysis would be finished at this point because the complete structure of the material is thought to be understood. However, if a complete automated statistical analysis of the spectral imaging data set is performed new features are brought to light.

For example, COMPASS finds that in addition to the three expected components (aluminum matrix, copper-enriched and iron-enriched particles - figure 2), there are also 3 unexpected components (figure 3). These components are found to be siliconrich, a tungsten-rich and an aluminum-copper-magnesium-silicon mixture.

XPhase analysis of the unexpected components determines that they are the only phases present in the material. The composition of each phase is measured and also the area fraction of each phase within the current field of view. In this example, the tungsten-enriched phase is only $0.3 \%$ of the area and the aluminum mixture phase is only $0.7 \%$ of the area, but both are expected to be a lower percentage in the whole sample.

With the additional information provided by the $\mathrm{X}$-ray microanalysis features, the engineer is able to determine if the original hypothesis of copper and iron particles affecting the properties is valid, or if the unexpected phases may be causing some problems.

COMPASS software finds image locations that have the same spectral fingerprint, with no assumptions about the chemical composition of the sample. Elemental phase maps show the spatial distribution of regions that have statistically similar spectral features (peak locations and relative peak heights) and cumulative spectra of these regions. Therefore, quantitative analysis of each elemental phase is now possible.

\section{Conclusion}

Phase determination using X-ray maps is burdened with problems, meaning that any conclusions drawn must still be verified. However, the COMPASS option on the NORAN System SIX automatically performs primary component analysis without intervention. Therefore, when using COMPASS the material analyst can be confident that any conclusions drawn from the results are accurate as COMPASS automatically suggests the correct answer, removing any bias about the elemental composition of the sample.

The COMPASS option provides an excellent means of identifying minor or unexpected components in a sample (the so-called 'needle in the haystack' components), locating distinct components where peak overlaps "hide" important regions, and in finding components down to the single pixel, even in very noisy data.

Using a collection of maps for input - which may be elemental count maps, elemental quantitative maps, or component maps from COMPASS - XPhase accurately identifies phases in the samples as shown in the aluminum alloy sample in figure 3 . This software option is particularly useful in cases where the analysis includes a large number of elements within each phase, or where the data contains only a few elements within a number of phases. Xphase enables the analyst extract data to provide a complete understanding of the material being analyzed.

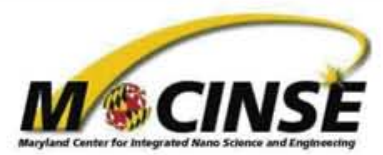

\section{Manager University Electron Microscopy Lab}

Institute for Research in Electronics and Applied Physics University of Maryland

The Lab Manager is responsible for the management and overall operations of a new university electron microscopy lab facility. The lab manager will support electron microscopy research in nanotechnology, materials science, chemistry, physics, and biology. A successful candidate should have expertise in a variety of transmission electron microscopy techniques used in the study of inorganic and organic/biological materials and in the use of the equipment to prepare samples for transmission electron microscopy such as polishing, ion milling, ultramicrotoming and safety issues in a microscopy laboratory. Familiarity with the areas of nanomaterials, electron tomography and imaging of beam sensitive materials is desirable. Facilities in the lab include a JEOL 2100F TEM, a 200kV LaB 6 TEM, a JEOL 8900 Superprobe. Responsibilities will include personnel and financial management of the facility. Strong communication, computing, and interpersonal skills are important. Education required: Master's degree in science or engineering required; $\mathrm{PhD}$ preferred. Salary will be commensurate with experience. Position will remain open until filled, for best consideration candidates should apply by December 15,2005 . The committee will allow additional time for people applying in January 2006

\section{Applications should be submitted electronically at: https://apra.umd.edu/search.jsp?ID=ENMA000003}

Chair of Search Committee: Professor Lourdes Salamanca-Riba, Materials Science and Engineering Dept., University of Maryland, College Park, MD 20742. 301-405-5220, riba@umd.edu

The University of Maryland is an equal opportunity/affirmative action employer. Women and minorities are encouraged to apply. 

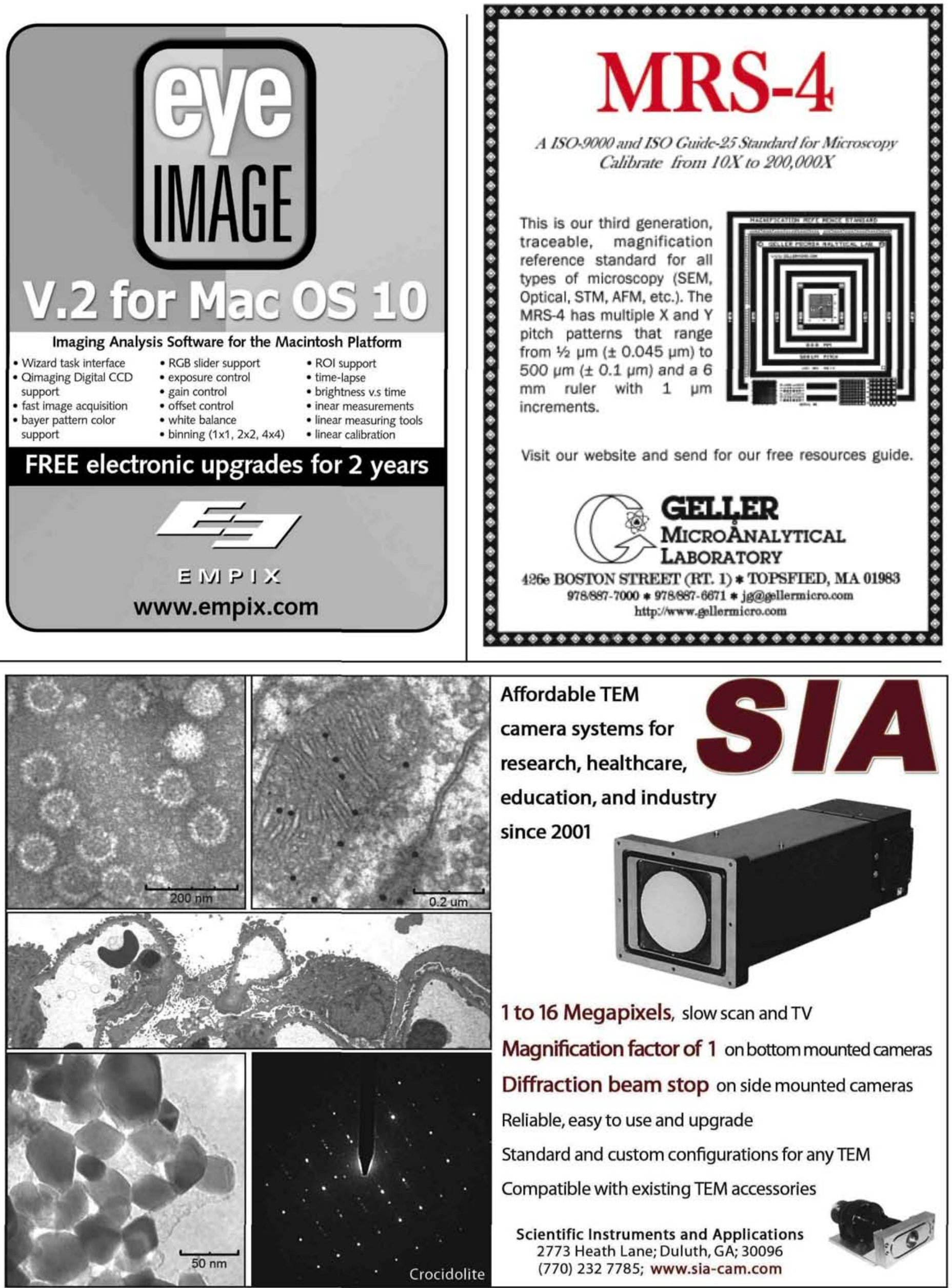

Affordable TEM
camera systems for
research, healthcare,

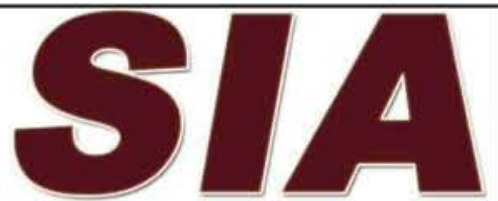
education, and industry since 2001

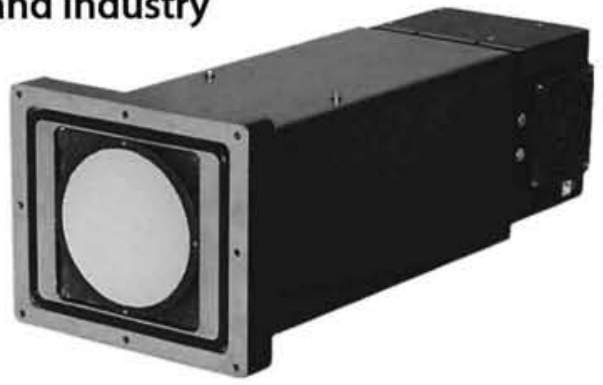

1 to 16 Megapixels, slow scan and TV

Magnification factor of 1 on bottom mounted cameras Diffraction beam stop on side mounted cameras

Reliable, easy to use and upgrade

Standard and custom configurations for any TEM

Compatible with existing TEM accessories

Scientific Instruments and Applications

2773 Heath Lane; Duluth, GA; 30096 (770) 232 7785; www.sia-cam.com

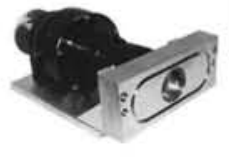

